\title{
Thermal spray ceramic coatings as friction surfaces of brake rotors
}

Septimiu Popa, Prof. Dr. Dr. R. Gadow, Prof. Dr. A. Killinger, Institute for Manufacturing Technologies

of Ceramic Components and Composites (IFKB), University of Stuttgart

This manuscript is not available according to publishing restriction.

Thank you for your understanding. 